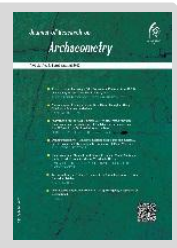

Book Review

\title{
Designing based on needs for the packaging, transportation and storage of human bones from archaeological excavations
}

\author{
Ramin Mohamadi Sefidkhani $^{1 \star}$, Zahra Rajabioun ${ }^{2}$ \\ ${ }^{1}$ M.A in Archaeometry, Institute of Archeology, University of Tehran, IRAN \\ ${ }^{2} \mathrm{MA}$ in Archaeology, University of Sistan and Baluchestan, IRAN
}

Received: 24/09/2019

Accepted: 21/11/2019

\begin{abstract}
Human burials from archaeological excavations are of great importance due to the available information on the history and culture of human societies. Today, in addition to studies of the burial form and the data in the grave, human bones have also shown the necessity of anthropological studies in the form of biological and archaeological studies to identify diets, types of diseases, Human Migration, etc that are based on the identification of the constituents of skeletons, stable isotopes, age and sex abundances, traumas, and so on.

Generally, Every year in archaeological excavations from Islamic sites to pre-historic sites, human and animal skeletal remains are found in various burials that their fate is as follows.

- Extraction of objects with bones, Burial record and bone re-burial.

- Fixed skeletons and their use as part of the site of museums in archaeological sites.

- Transfer of discovered skeletons to museums using blocking techniques.

- Takings of skeletons in separate pieces and transfer to museum repositories for display or storage for future scientific studies.

The last option has attracted a lot of attention over the last few years. However, transportation conditions and improper maintenance can cause them to be destroyed or re-buried. In fact, data from the time of discovery require to take proper packaging, safe transportation, storage and maintenance in suitable environmental conditions, and the simple and quick ability to move in crisis management situations; Also, providing researchers with secure, orderly and directional data is of high importance.

This note attempts to provide a practical solution with regard to the condition of the taken bones from sites, initial transportation and temporary on-site storage, and ultimately transportation to museum reservoirs and their maintenance. These solutions allow the temporary and permanent transfer and maintenance of skeletons, along with the convenience of inspection and study, and the safe maintenance or transportation for good. These solutions make it possible to transport and maintain skeletons with the convenience of review and study, and permanent maintenance or safe transport. For this purpose, considering the largest bones dimensions discovered in archaeological excavations, and the possibility of dividing human bones segments into matched groups, designing separable boxes and reassembly to hold a human skeleton. The box is designed in four separate floors with a total height of 66 , width 33 , and length of $57 \mathrm{~cm}$, which are connected by sliding frame and cap and side clamps (picture1,2):
\end{abstract}

- The floor is $12 \mathrm{~cm}$ high with three sections designed to separate the bones of the thighs

*Corresponding author: ramin.mohamadi@rocketmail.com 
and legs.

- The second part is $12 \mathrm{~cm}$ high with three sections for left and right hands and forearm placement.

- The third part, $12 \mathrm{~cm}$ high, consists of three parts: ribs, spines and jaws.

- The fourth part, $26 \mathrm{~cm}$ high, is the largest part of the human skeleton, including the skull, pelvis and shoulders.

- The cover is attached to the box frame which is a sliding frame to accommodate the boxes and The cover section is intended to accommodate documentation and sample storage(picture 2. 3) .

Considering the cost of the transport and temporary picking up to the workshops and warehouses museums, and often opening and re-packing in museums tanks that require several different packaging tools, the design can be quite justifiable by mass production would probably be lower than the final price. Proposed materials for this design can be polyester materials for the external sector, blends of polyurethane foam with polyester coating for the interior sector. Despite its lightweight and durable of the final product of the design, In the case of mass production the final price of the product is reduced significantly. And if you use the box from data discovery to shipping, storage, and direct access to the various bony sections, it will provide good economic and practical justification. On the other hand, the box can be attached to be fixed and installed in museums' tanks, which also improves how data maintenance is (picture 4). This box can be useful for animal skeletons that have significant abundance in archaeological excavations in different areas, as well as economically justifiable. 


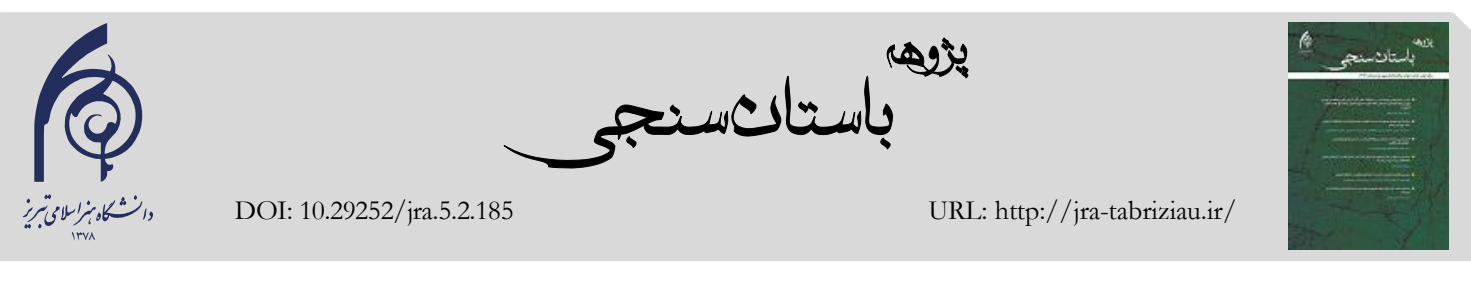

ي يِادداشت فنى

\section{طراحى مبتنى بر نياز در راستاى بستهبندى، حملونقل، ذخيره

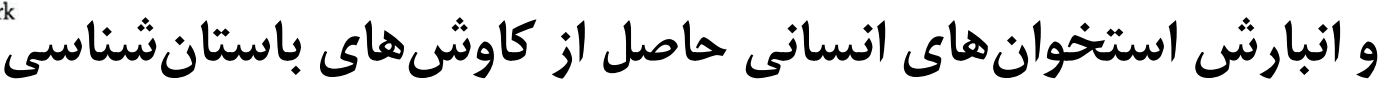

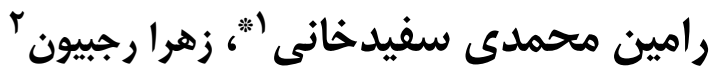

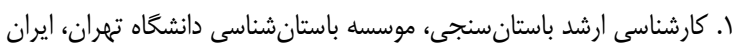

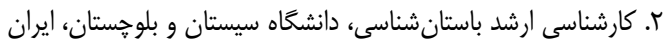

ميانرشتهاى در رشد و گسترش بسـيارى از شـاخههـاى باستانشناسى شاهد هستيم متأسفانه استفاده از دادههاى

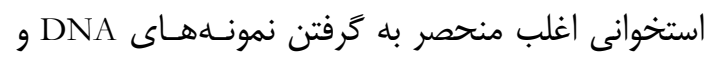

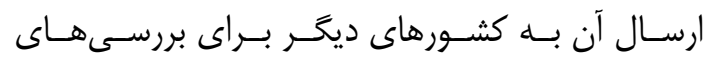
آزمايشكاهى و زنتيكى است.

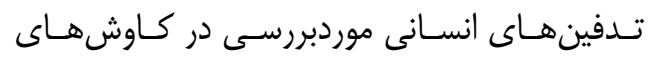
باستانشناسى بهواسطه اطلاعات ذخيرهشـده از تـاريخ و

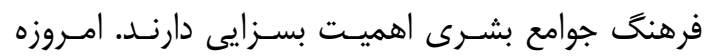

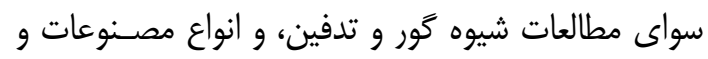
بقاياى آن، استخوانهاى انسانى نيز ضـرورت مطالعات

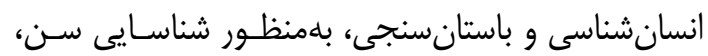

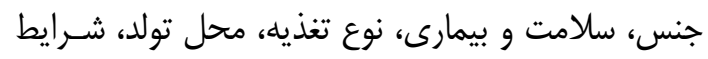

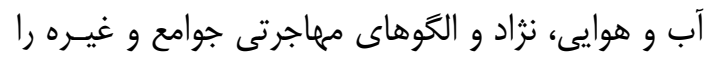

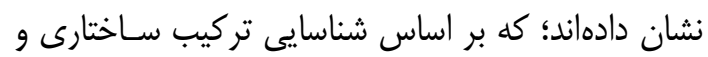

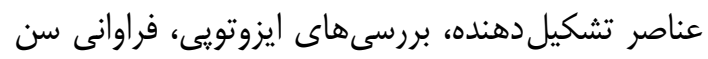

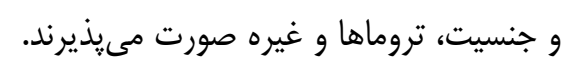

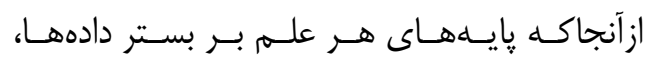

هدف در باسـتانشناسـ، شـناخت كزشـته و خاسـتخاه

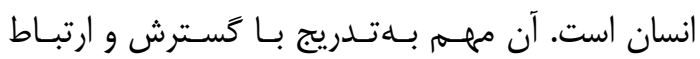

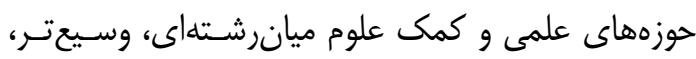

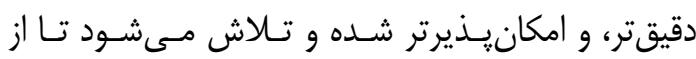
يافتههاى كاوش شده حداكثر اطلاعات استخراج كَردد.

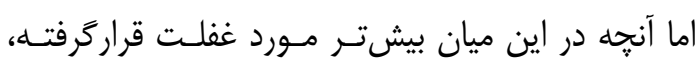

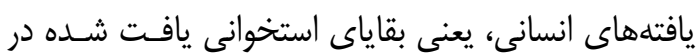

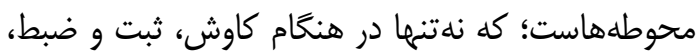

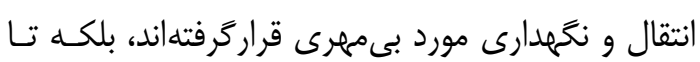

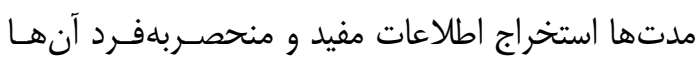

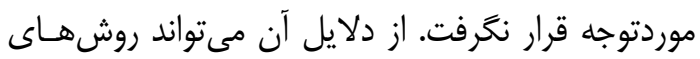

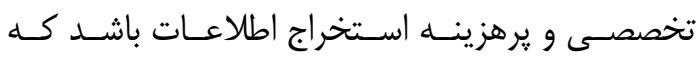

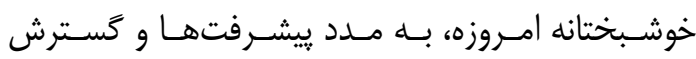

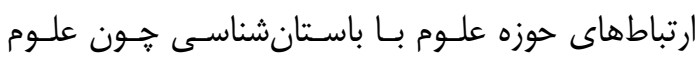
زنتيك و بيومولكولى دسترسى به متخصصـين و ابزارهـا

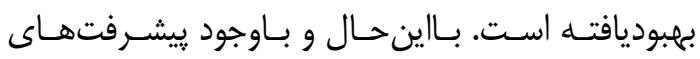

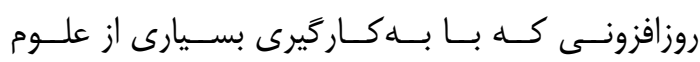

" مسئول مكاتبات: تهران، خيابان انقلاب اسلامى، خيابان قدس، خيابان يورسينا، پِلاك سا، موسسه باستانشناسى دانشكاه تهران.

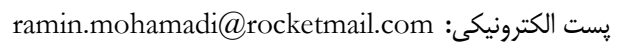
C) حق نشر متعلق به نويسنده(كان) است و نويسنده تحت مجوز Creative Commons Attribution License به مجله اجازه مىدهد مقاله خاب شده را با ديخران به اشتراك بكذارد منوط بر اينكه حقوق مؤلف اثر حفظ و به به انتشار اوليه مقاله در اين مجله اشاره شود. 
باستانى، حمل ونقل اوليه و نكَهدارى موقـت در محـل، و

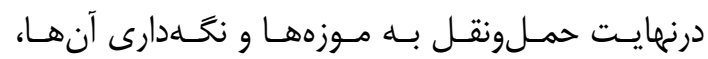

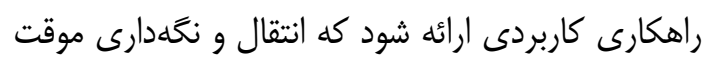

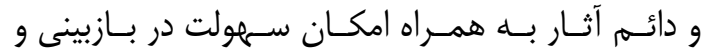

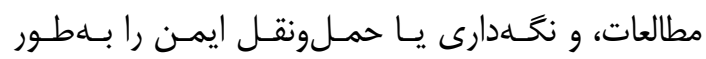

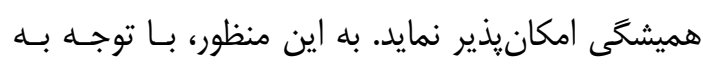

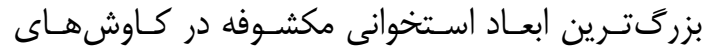

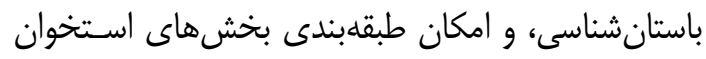

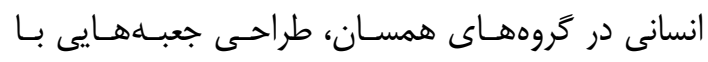

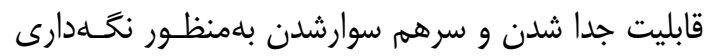

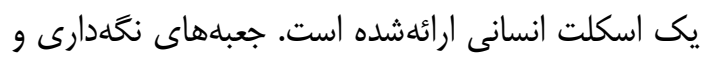

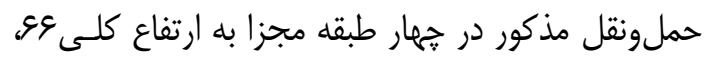

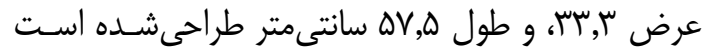

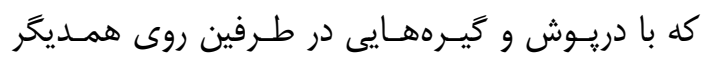
متصل مىشوند (تصوير ا و r r):

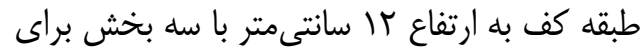

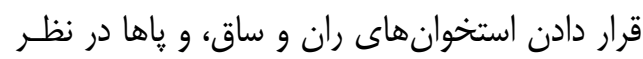
كرفتلشده است. طبقه دوم نيز بهمانند طبقه كـف بـا همـان ارتفـاع

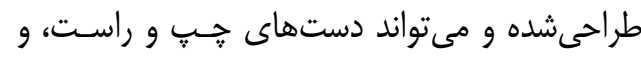

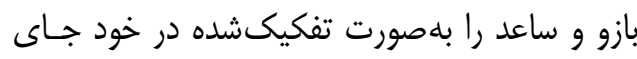

قسمت سوم با همان ارتفاع در دو قسـمت، بخـش درم

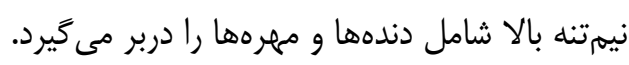

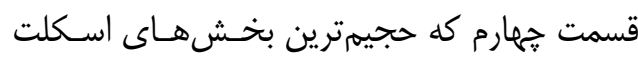

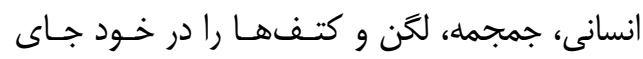

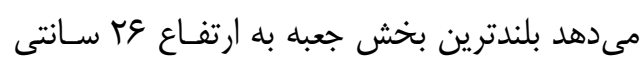
متر است.

دريوش به همراه جِهار خوب جعبه متصل بههم است

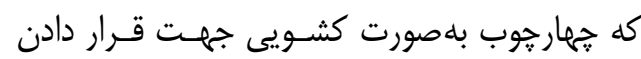
جعبهها تنظيمشده و در قسمت دريوش بـراى قرار

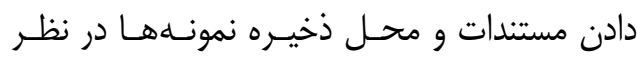
كرفتهشده است (تصوير ץ و بَ).

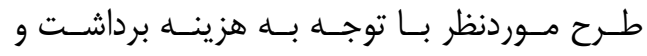

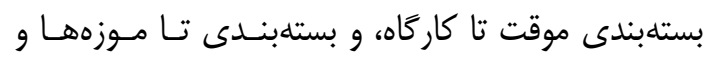

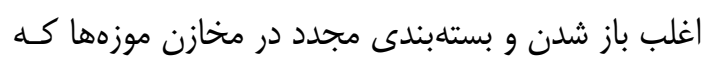

طبقهبندى آنها، و آزمايش و بررسى آن دادههـا اسـتوار

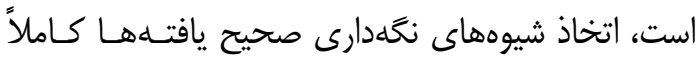

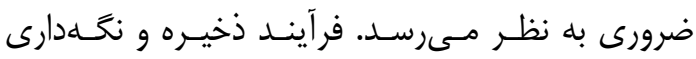

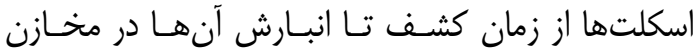

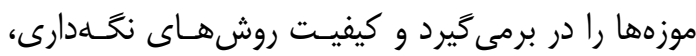

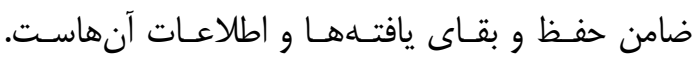
هملساله در كاوشهاى علمسى باسـتانشناسى بقايساى

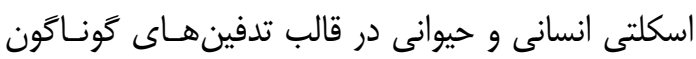

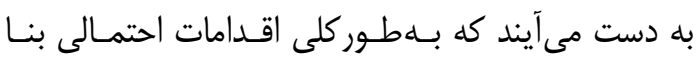
بلهرورت شرايط حاكم نسبت به هنين يافتههايى بدين

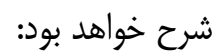

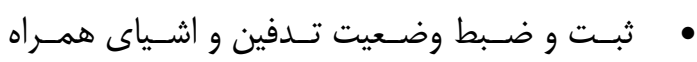
استخوانها، و بازتدفين مجدد استخوانها

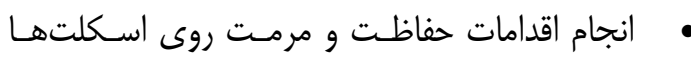

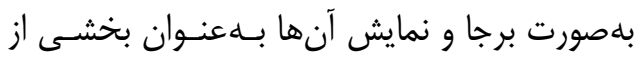

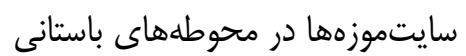
انتقال اسكلتهاى مكشوفه به بايخاههاى مطالعات

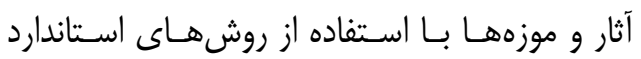
انتقال و بستهبندى از قبيل بلوكهبردارى

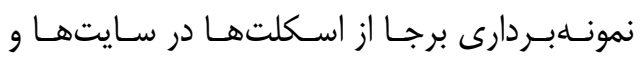
بازتدفين آنها برداشت اسكلتها بلهورت قطعات مجزا و انتقال

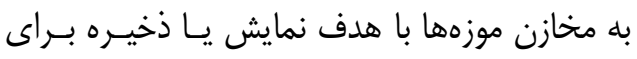
انجام مطالعات علمى آتى......

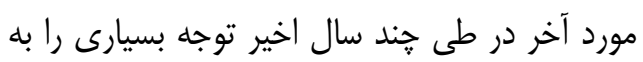

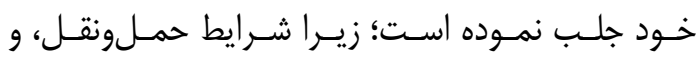

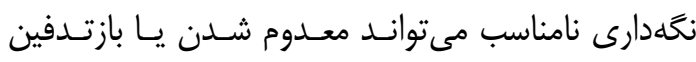

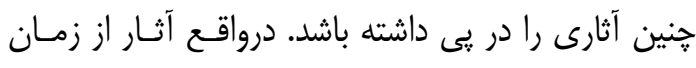

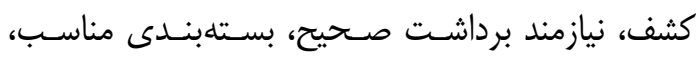

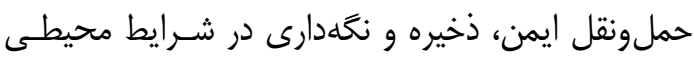

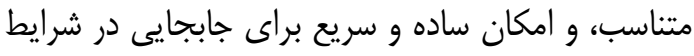

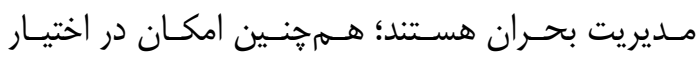

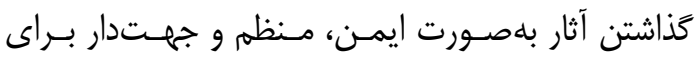
يزوهشكران حوزههاى مربوطه نيز حائز اهميت است.

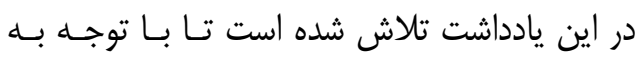

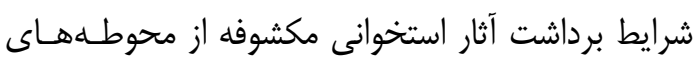


جعبه مذكور مىتواند در سازههاى نصبشده در مخـازن موزهها متصل و ثابت شود كه نحوه نخحددارى آثار را نيـز مئز بعبود مىبخشد( تصوير أ). از اين نوع جعبلها مسىتـوان

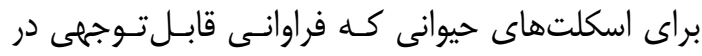

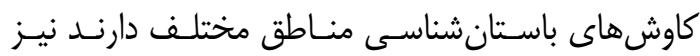
طراحى، توليد و استفاده نمود.

\section{سباسگَزارى}

نغارندكان از راهنمـايىهـاى خـانم دكتــر الهـام فرنـام،

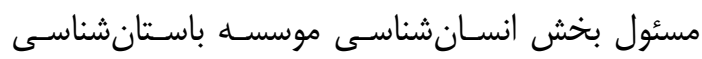

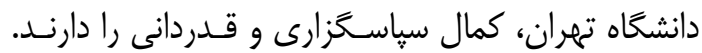

نياز به جُند ابزار مختلـف دخيـل در بسـتهبنـدى اسـت،

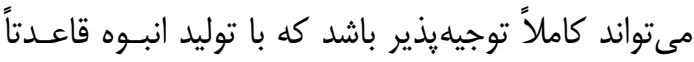

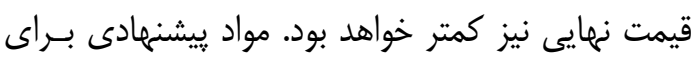

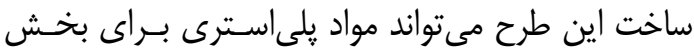

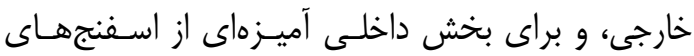
يلىاورتانى به همراه روكش يلى استرى باشد كه علىرغم برهم

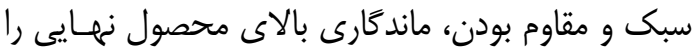

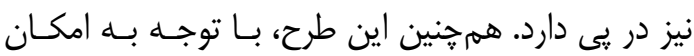

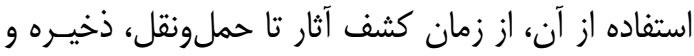

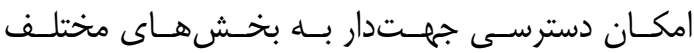

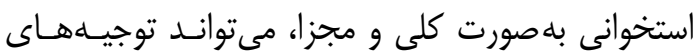
اقتصادى و كاربردى مناسـبى را نشـان دهـد. از طرفى مئى

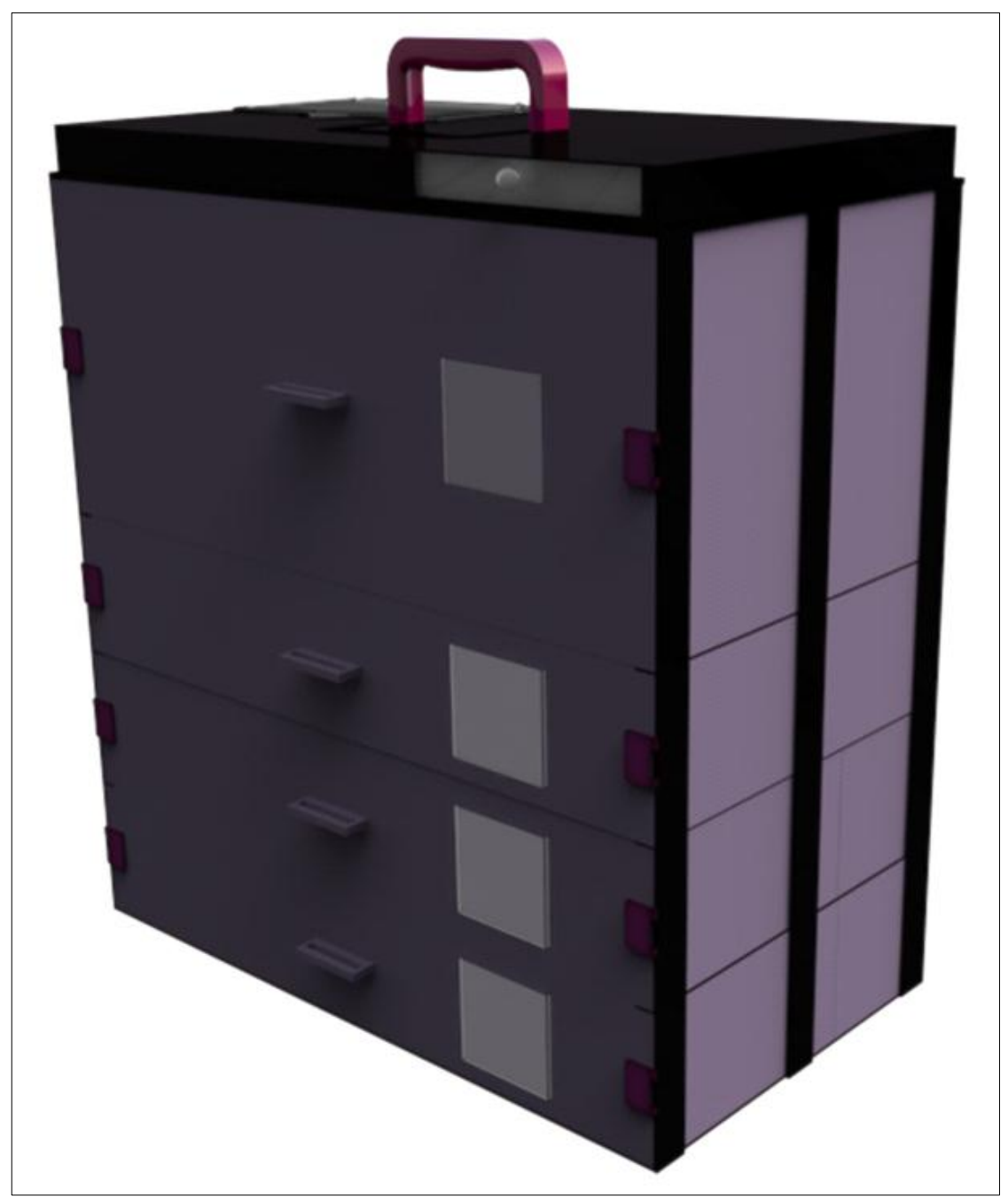

شكل ا: نمايى از جعبه طراحى شده براى اسكلت انسانى

Fig.1: Box designed to accommodate the human skeleton 


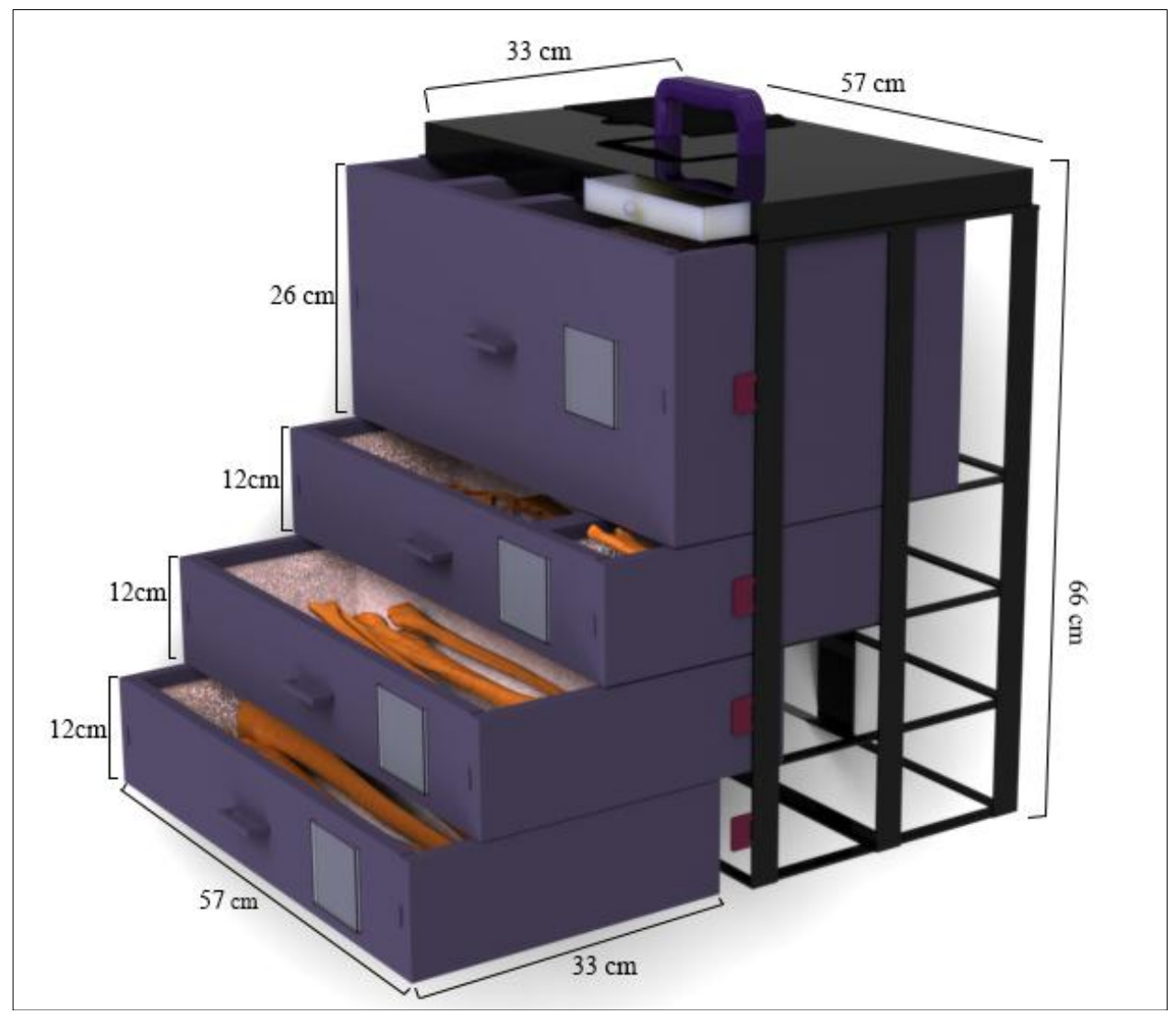

شكل ז: نمايى ديخر از جعبه مورد طراحى براى اسكلت انسانى و ابعاد آن

Fig.2: Box dimensions designed for human skeleton placement

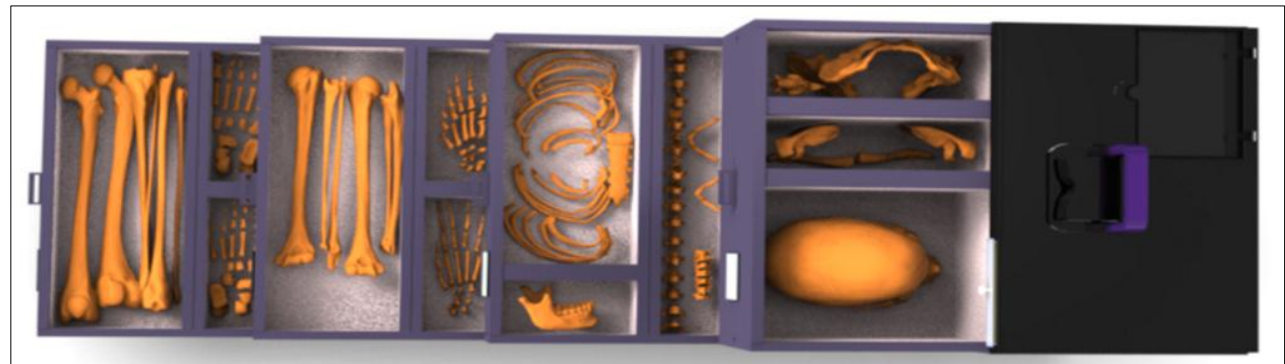

شكل سّ: نماى بالا از جعبه مورد طراحى براى اسكلت انسانى

Fig. 3: Interior of the designed box 


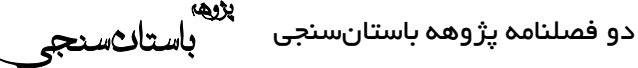

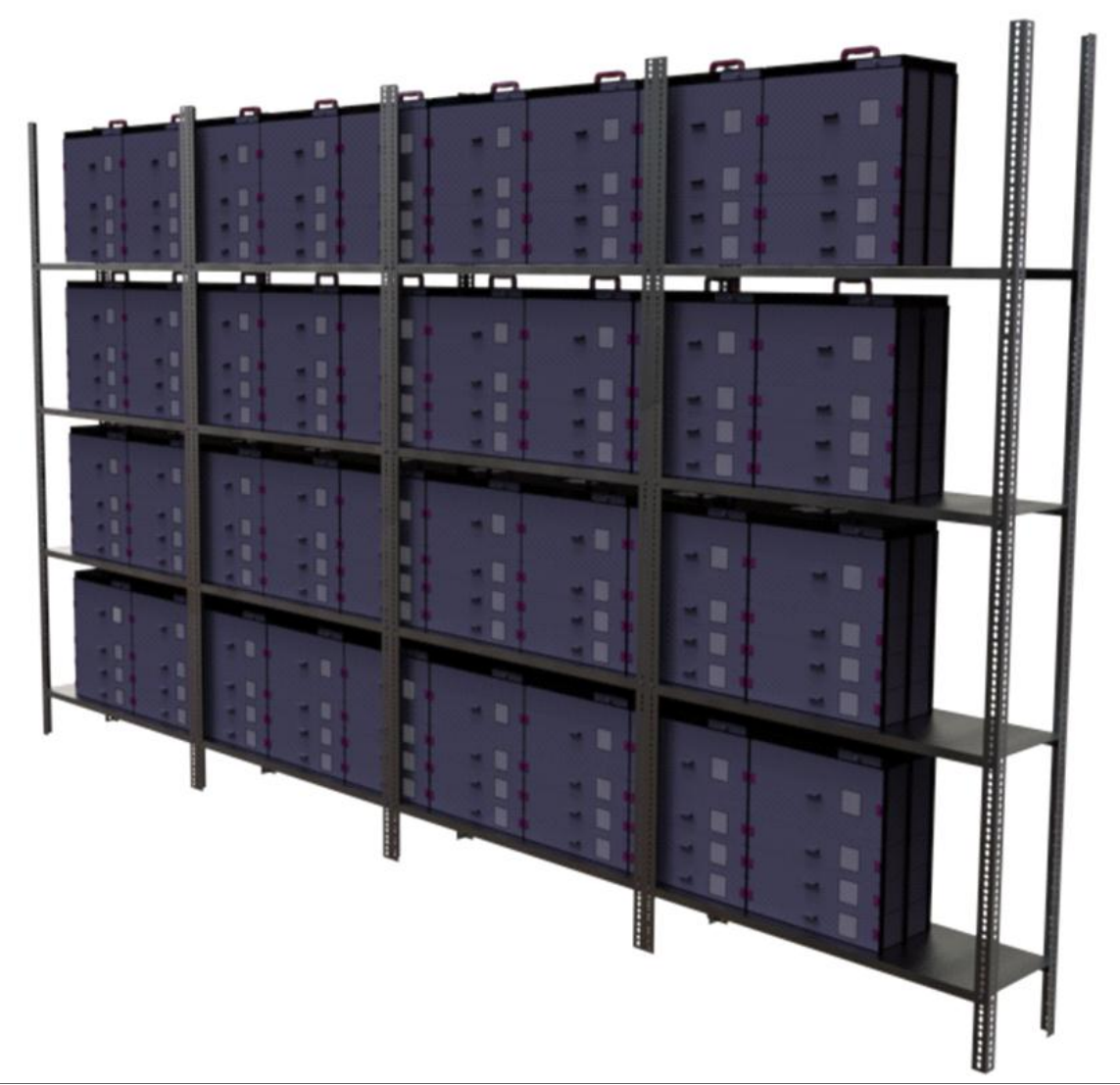

شكل ثا: نمايى از نحوه قرارگيرى جعبههاى مورد طراحى براى اسكلت انسانى در مخازن موزهها

Fig. 4: Putting boxes in museum tanks 